\title{
Mechanical Ventilation: Physiology and Practice, Second Edition
}

\author{
John W. Kreit (Author), John A. Kellum (Series Editor). Oxford University Press 2018; \\ \$71.50 CAD (paperback); 219 pages. ISBN: 9780190670085
}

\author{
Ricardo Teijeiro-Paradis, MD · Lorenzo Del Sorbo, MD (1)
}

Received: 10 December 2018/Revised: 19 January 2019/Accepted: 30 January 2019/Published online: 12 February 2019

(C) Canadian Anesthesiologists' Society 2019

Mechanical ventilation is a mainstay of treatment for respiratory failure and the most frequent indication for admission to an intensive care facility. Hence, the theory, function, physiology, application of mechanical ventilation, and relevant guidelines are of fundamental clinical importance and are the focus of Mechanical Ventilation: Physiology and Practice, Second Edition. Dr. John W. Kreit, the single author of all chapters of this book, is a senior clinician-educator and Professor of Medicine and Anesthesiology in the Division of Pulmonary, Allergy, and Critical Care Medicine, University of Pittsburgh School of Medicine. Dr. Kreit is a critical care specialist with interests in medical education and the diagnosis and treatment of respiratory failure and mechanical ventilation. Dr. John A. Kellum, also a critical care specialist, is a member of the Division of Pulmonary, Allergy, and Critical Care Medicine, University of Pittsburgh School of Medicine and is the Editor of the Pittsburgh Critical Care Medicine Series, to which this book belongs.

The book is organized into three logically structured, similarly sized sections: Essential Physiology, The Mechanical Ventilator, and Patient Management. Each section contains three to ten chapters ranging in length from five to 30 pages. The text is easy to read and blends complex concepts and terminology with clinical examples to facilitate comprehension. The chapters contain informative illustrations, graphs, and tables that aid in the understanding and flow of reading. At the end of each chapter, three to five references are provided as additional reading suggestions.

The first section of Mechanical Ventilation: Physiology and Practice provides a comprehensive overview of the basic

R. Teijeiro-Paradis, MD - L. Del Sorbo, MD ( $\varangle)$

Interdepartmental Division of Critical Care Medicine, University

Health Network - Sinai Health System, University of Toronto,

Toronto, ON, Canada

e-mail: lorenzo.delsorbo@uhn.ca physiology relevant to mechanical ventilation. The included chapters are "Respiratory Mechanics," "Gas Exchange," and "Cardiovascular-Pulmonary Interactions." The chapters explain key concepts regarding the interface between the mechanical ventilator and the patient. The use of simple examples, such as "straws and balloons," facilitate understanding basic principles. There is appropriate use of graphs and figures to aid comprehension without oversimplifying concepts.

Section Two is devoted specifically to the mechanical ventilator. The included chapters are "Instrumentation and Terminology," Ventilator Modes and Breath Types," and "Ventilator Alarms - Causes and Evaluation." The chapter "Ventilator Modes and Breath Types" provides practical and essential information regarding the ventilation mode and type of breath delivery in a simple manner. The information is particularly useful as there is no universal nomenclature for this area of expertise. Currently, there are multiple ventilator modes and breath types which can be overwhelming to those not expert in this field. The summary table and the use of mathematical models and graphs greatly facilitate comprehension. The chapter concerned with ventilator alarms and problem troubleshooting is practical and includes clear illustrations and tables that summarize the key points.

Section Three focuses on ventilator-patient interactions. The chapters include "Respiratory Failure and Indications for Mechanical Ventilation," "How to Write Ventilator Orders," "Physiological Assessment of the Mechanically Ventilated Patient," "Dynamic Hyperinflation and IntrinsicPositiveEndExpiratory Pressure," "Patient-Ventilator Interactions and Asynchrony," "Acute Respiratory Distress Syndrome (ARDS)," "Severe Obstructive Lung Disease," "Right Ventricular Failure," "Discontinuing Mechanical Ventilation," and "Noninvasive Mechanical Ventilation."

A review of the basic physiology underlying hypoxemic respiratory failure in the chapter "Respiratory Failure and 
Indications for Mechanical Ventilation" is followed by a consideration of the physiological monitoring options, including oxygenation indices and capnography, as well as a review of respiratory mechanics, in the chapter "Physiological Assessment of the Mechanically Ventilated Patient." A unique chapter, "How to Write Ventilator Orders", provides recommendations on how to initiate mechanical ventilation which would be particularly useful to those with limited expertise with this responsibility.

The chapter "Acute Respiratory Distress Syndrome (ARDS)" is noteworthy as it provides highly useful information for those caring for this most challenging patient. The chapter begins with a detailed, comprehensive review of the defnition and pathophysiology of ARDS, followed by a discussion of the relevant issues regarding mechanical ventilation management. The chapter emphasizes the underlying cardiopulmonary interactions with regard to changes in hemodynamics and ventilation-perfusion mismatch and how monitoring oxygen delivery (rather than oxygen saturation or content) should be the priority that is individualized for each patient. Mechanical ventilation management is also thoroughly reviewed, including the appropriate inspiratory oxygen concentration, tidal volume, and respiratory rate; initiation and titration of positive endexpiratory pressure; and recruitment maneuvers. Ventilatorinduced lung injury is well covered, and the chapter closes with a summary of the evidence-based role for ancillary treatment options for ARDS, including prone-position ventilation, neuromuscular blockade, inhaled vasodilators, and extracorporeal membrane oxygenation.

The last two chapters of Section Three, "Discontinuing Mechanical Ventilation" and "Noninvasive Mechanical Ventilation," focus on weaning the patient from mechanical ventilation, with consideration of invasive and noninvasive control of the airway. A crucial element reviewed in "Discontinuing Mechanical Ventilation" is the systematic approach to the patient who fails a spontaneous breathing trial and the subsequent interventions that can be applied before repeating any further spontaneous breathing trials.

In summary, Mechanical Ventilation: Physiology and Practice, Second Edition provides information essential for managing mechanically ventilated patients. The book's organization and structure, including the use of explanatory figures and illustrations, help the reader understand information that can be complex and dense. Nevertheless, the liberal reliance on mathematical equations and principles of physics to explain the scientific underpinnings of mechanical ventilation could be challenging for the casual reader. To improve future editions of this book, we recommend including summarizing tables with the elemental key points at the end of each chapter and complementing the information with a link to active webbased simulations or examples to illustrate the most important principles.

We strongly recommend this book to intensivists, anesthesiologists, surgeons, and respiratory therapists caring for mechanically ventilated patients. Moreover, the book would be a useful addition to any Department of Anesthesia reference library as anesthesia machine ventilators are becoming increasingly more complex in design, with an array of ventilation settings and parameters.

Competing interests The authors declare that they have no competing interests.

Editorial responsibility This submission was handled by Dr. Steven Backman, Associate Editor, Canadian Journal of Anesthesia.

Funding This article did not receive sponsorship for publication.

Publisher's Note Springer Nature remains neutral with regard to jurisdictional claims in published maps and institutional affiliations. 